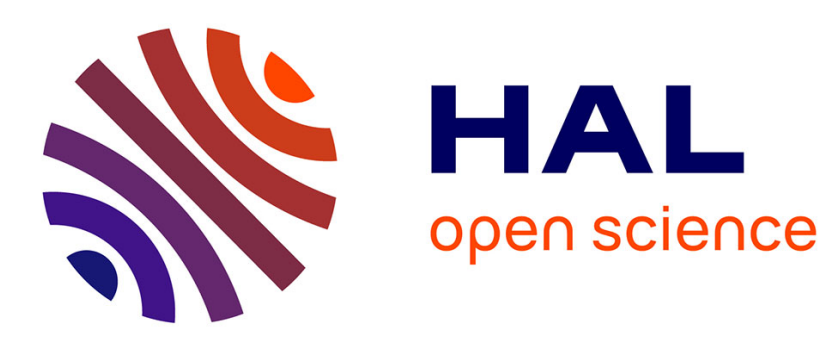

\title{
An Overview of Channel Coding For Underwater Acoustic Communications
}

Joël Trubuil, André Goalic, Nicolas Beuzelin

\section{To cite this version:}

Joël Trubuil, André Goalic, Nicolas Beuzelin. An Overview of Channel Coding For Underwater Acoustic Communications. MILCOM 2012: IEEE Military Communications conference, Oct 2012, Orlando, United States. pp.1 - 7. hal-00828185

\section{HAL Id: hal-00828185 https://hal.science/hal-00828185}

Submitted on 30 May 2013

HAL is a multi-disciplinary open access archive for the deposit and dissemination of scientific research documents, whether they are published or not. The documents may come from teaching and research institutions in France or abroad, or from public or private research centers.
L'archive ouverte pluridisciplinaire HAL, est destinée au dépôt et à la diffusion de documents scientifiques de niveau recherche, publiés ou non, émanant des établissements d'enseignement et de recherche français ou étrangers, des laboratoires publics ou privés. 


\section{An Overview of Channel Coding For Underwater Acoustic Communications}

\author{
Joël Trubuil, André Goalic \\ Institut TELECOM; TELECOM Bretagne; \\ UMR CNRS 3192 Lab-STICC \\ BP 83818, 29238 Brest Cedex 3, France \\ joel.trubuil@telecom-bretagne.eu
}

\author{
Nicolas Beuzelin \\ G.E.S.M.A. BP 42, \\ 29240 Naval Brest, France \\ Nicolas.beuzelin@dga.defense.gouv.fr
}

\begin{abstract}
The TRIDENT (TRansmission d'Images et de Données EN Temps réeel) project was launched a few years ago by GESMA (Groupe d'Etudes Sous Marine de l'Atlantique). The initial objectives were to develop a multiple-rate underwater acoustic link for images, text and data transmission. Later, the speech option was added, and more recently channel coding options have been introduced to the TRIDENT platform. Convolutional codes (CC) and Reed Solomon (RS) block codes were then checked, but these simple codes were not able to significantly improve the Bit Error Rate (BER) at the channel decoding output. For this reason, GESMA decided to introduce turbo codes options, more specifically the Reed Solomon Block Turbo Codes (RS-BTC), to enhance the channel decoding efficiency. The data transmitted are interleaved and frame recovery is performed in reception. After system validation in static conditions, in the Penfeld river in Brest, France, sea trials were conducted in the Bay of Brest in dynamic conditions. This paper presents various possibilities offered by the platform for images and speech transmissions and the RS-BTC channel coding options.
\end{abstract}

Keywords- Underwater acoustic communication; BER, Convolutional codes (CC); Reed Solomon (RS) block codes; Reed Solomon Block Turbo Codes (RS-BTC).

\section{INTRODUCTION}

The initial objective of the TRIDENT project (TRansmission d'Images et de Données EN Temps réel) was to equip Autonomous Underwater Vehicles (AUVs) with an acoustic communication link. This system had to be able to transmit continuous information such as images, speech, data and text. The TRIDENT system (Fig. 1) [1] can use four carrier frequencies $(11.2,17.5,20.0$ and $34 \mathrm{kHz})$ with a Quadrature Phase Shift Keying (QPSK) modulation and different bit rates, ranging from 2.8 to $23.3 \mathrm{kbps}$. Multipath propagation, Doppler effect and noise bring a lot of perturbation on underwater acoustic communication, such as time dispersion and variability to the received signal. It should be noted that carrier frequencies and available bandwidths are much lower than those of radio communication channels. A blind spatiotemporal equalizer [2] is used to reduce these various perturbations. GESMA also aimed to increase the link's reliability with the goal of lowering the Bit Error Rate (BER) from $10^{-2}$ to $10^{-4}$. In order to do so, channel coding was integrated into the system. Two kinds of error correcting schemes had been tested, namely Convolutional Codes (CC) and Reed Solomon (RS) block codes [3-4], both hard and soft decoding versions. These two channel coding schemes did not provide an adequate level of correction. To improve the symbol correction with a higher code rate, Reed Solomon Block Turbo Codes (RS-BTC) were recently tested, in both static and moving conditions. The iterative decoding process based on the Chase-Pyndiah [5-6] algorithm uses a soft decoding version with eight iterations. Berlekamp [7] and Chien algebraic algorithms are also used to correct symbols during the iterative process. Synchronization words are wisely included in the coded frame to synchronize the interleaver and recover the information frame at the receiver. A differential coding/decoding is used to solve phase ambiguities.

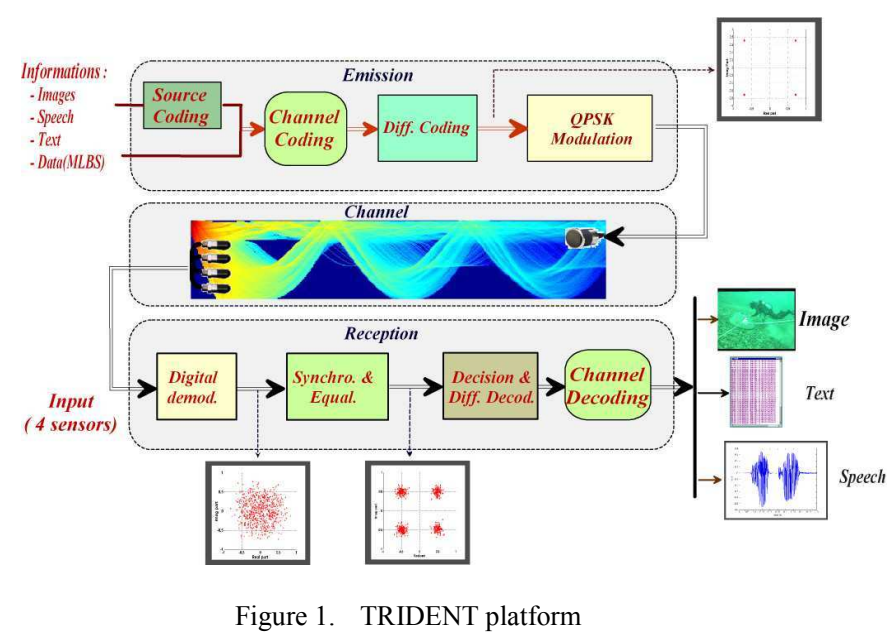

This paper provides an overview of the high data rate acoustic link. Firstly, we present the TRIDENT platform and channel coding strategies, including the $\mathrm{CC}$ and $\mathrm{RS}$ options, with the RS-BTC extension. Then we present the sea trials and show the acoustic communication results in the 2.8 to $14 \mathrm{kbps}$ range, applied both to images and low bit rate speech $(2400$ bps) transmissions. 


\section{CHANNEL CODING STRATEGIES}

The signal is received on four hydrophones, coming from the same source of emission and demodulated. Timing and carrier recovery, equalization are then processed before making the decision upon the transmitted symbol. The equalizer is a multiple-input self-adaptive Decision Feedback Equalizer. This equalizer is able to run in one of two modes: a convergence or starting mode, in which the equalizer is self-controlled, and a tracking mode, where it is controlled by its own decisions. Switching between the two modes is performed in an automatic and reversible way by comparing the Mean Square Error (MSE) with a threshold.

In order to improve the acoustic link, channel coding may correct remaining errors. The goal is to decrease the BER from $10^{-2}$ down to $10^{-4}$. Different channel coding have been tested, including Convolutional Codes (CC), Reed Solomon (RS) block codes and Reed Solomon Block Turbo Codes (RS-BTC).

\section{A. Convolutional Codes (CC)}

Convolutional Codes are generally specified by three parameters $(n, k, m)$, where $n$ is the number of output bits, $k$ represents the number of input bits and $m$ corresponds to the number of memory registers. The code rate $R=k / n$ is a measure of the code efficiency. Commonly $k$ range from 1 to 8 and $n$, from 2 to 10 . Usually CC is specified by parameters $(n$, $k, l)$, where $l$ is the code constraint length defined by $l=k(m-l)$ and represents the number of bits in the encoder memory that affect the $n$ output bit generation. Two Convolutional Codes were evaluated, namely $\mathrm{CC}(7,5)$ and $\mathrm{CC}(35,23)$.

The decoding process uses the Viterbi algorithm with a trellis representation, with 4 or 16 states according to the CC coder. Decoding is performed with hard or soft options. Hard decoding uses only binary values, whereas the soft option uses real values coming from the output equalizer. In order to close the trellis, zero tail bits are added at the end of the frame, respectively two for $\mathrm{CC}(7,5)$ and four for $\mathrm{CC}(35,23)$. Synchronization words (respectively set to 16 and 14 bits) are included to retrieve the 54 bits of data (speech frame). Whatever the CC in use and including the trellis tail bits, the information frame is a 72-bit length. With a code rate $R$ equal to 0.5 , the emitted coded frame is 144 bits in length. The whole correcting rate is then 0.375 . After the coding process, the synchronization word is 32 bits (respectively 28) long.

CC $(7,5)$ is defined by (1) and (2) and its synoptic is represented in Fig. 2. The scheme for CC $(35,23)$ is shown in Fig. 3 and this code is defined by (3) and (4).

$$
\begin{gathered}
s_{1}(x)=\left(1+x+x^{2}\right) e(x) . \\
s_{2}(x)=\left(1+x^{2}\right) e(x) . \\
s_{1}(x)=\left(1+x+x^{2}\right) e(x) . \\
s_{2}(x)=\left(1+x+x^{2}+x^{4}\right) e(x) .
\end{gathered}
$$

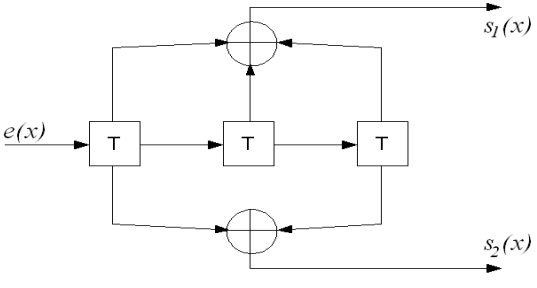

Figure 2. Convolutional Code $(7,5)$

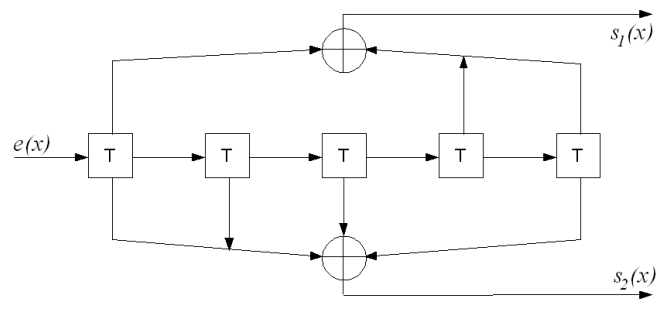

Figure 3. Convolutional Code $(35,23)$.

\section{B. Reed Solomon (RS) block codes}

Reed Solomon codes $(n, k, t)$ are cyclic codes, built from $n$ symbols with a maximum of $n=q-1$, where $q$ is the number of elements in the Galois field $\left(G F^{q}\right)\left(q=2^{n}\right)$ and $t$ is the power correcting code, so the number of control symbols is $2 t . k=n$ $2 t$ represents the number of information symbols that can be transmitted. The decoding process can be hard or soft. Based on the Chase algorithm, the soft decoding process uses the Berlekamp and Chien algorithms to correct received symbols. In our case, we are looking for four unreliable bits and checking among 16 possible code words. Two Reed Solomon codes were evaluated, namely $\operatorname{RS}(31,29)$ and RS $(31,27)$. A synchronization word $S$ is included in the frame to retrieve the data (speech frame) at the receiver side. According to RS power correcting code $t, S$ is 29 bits in length $(t=1)$ and $27(t$ $=2$ ). To match the chosen RS code size, the information matrix processes over two speech frames (108 bits). The whole correcting rate $R$ is equal to 0.7 of whatever the correcting power is. The Underwater Acoustic (UWA) channel is characterized by burst errors coming from multipath and noise. Writing and reading matrix strategies are likely to improve the correcting process by working like an interleaver.

\section{Reed Solomon Block Turbo Codes (BTC-RS)}

The concept of product codes is a simple and efficient method to construct powerful codes with a large minimum Hamming distance, $\delta_{p}$, using conventional linear block codes [6]. Let us consider a systematic linear block code $C$ having parameters $(n, k, \delta)$ as illustrated in Fig. 4. The parameters $n$ and $k$ stand for the code length and the number of information symbols, respectively. The product code $P=C \times C$ is obtained by placing $k^{2}$ information bits in a matrix of $k$ rows and $k$ columns and encoding the $k$ rows and $k$ columns using the code $C$. It can be shown that all $n$ rows and all $n$ columns are code words of $C$. Furthermore, the parameters of the resulting product code $P$ are given by $n_{p}=n^{2}, k_{p}=k^{2}, \delta_{p}=\delta^{2}$. The code 
rate $R_{p}$ is given by $R_{p}=R^{2}$ with $R=k / n$. Thus, it is possible to construct powerful product codes based on linear block codes, such as RS codes. Working in the Galois field $G F^{q}$, RS codes are a class of linear cyclic block codes that have capabilities for multiple error detection and correction.

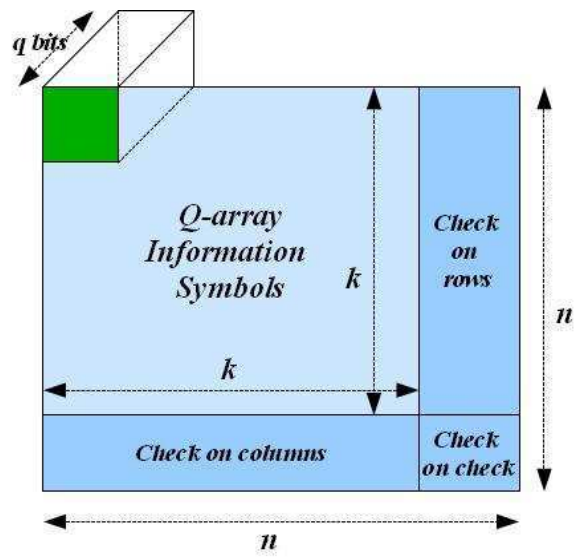

Figure 4. RS product codes with Q-ary symbol concatenation

Product code decoding involves sequential decoding of rows and columns using a Soft Input Soft Output (SISO) decoding algorithm (Fig. 5). The block turbo decoding process repeats this soft decoding for several iterations. Each decoding process computes soft information $R_{(i t+l)}^{\prime}$ from the channel received information $R$ and the information computed in the previous half iteration, $R^{\prime}(i t)$. The extrinsic information $W_{(i t)}$ is obtained by subtracting the soft input information $R^{\prime}(i t)$ from the

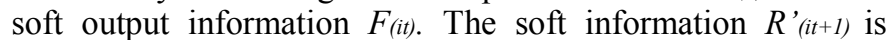

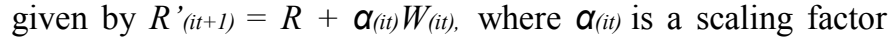
that reduces the effect of the extrinsic information in the soft decoder during the first decoding steps.

In 1972, Chase proposed algorithms that approximate the optimum Maximum-Likelihood (ML) decoding of block codes with low computing complexity and small performance degradation. In 1994, Pyndiah et al. presented a new iterative decoding algorithm for decoding product codes, based on the iterative SISO decoding of concatenated block codes.

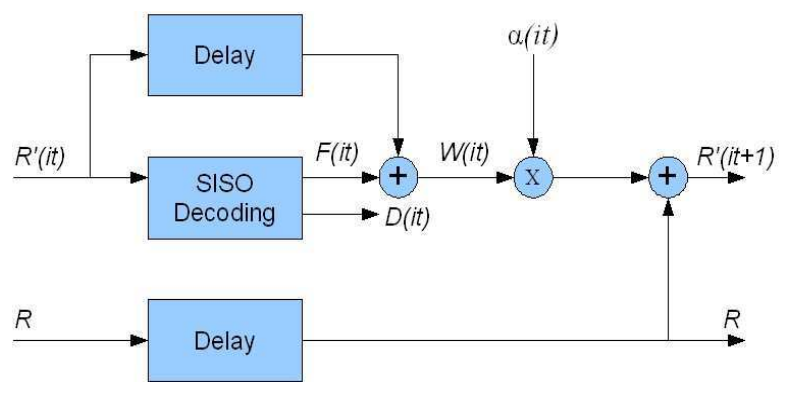

Figure 5. Iterative block turbo decoding principle

So far the TRIDENT system has used the $\operatorname{RS}^{2}(31,29,1)=$ $\operatorname{RS}\left(n=31=2^{q}-1, k=29=n-2 t, t\right)$ BTC channel coding $(q=5$, $t=1 ; t$ being the correcting power). Three different synchronization words $S_{i}(i \in\{1,2,3\})$ are included to retrieve the frame, at the receiver side. $S_{1}$ (or, respectively, $S_{2}$ or $S_{3}$ ) is used to detect the frame start (respectively middle or end frame). The emitted coded frame, with $S_{i}$ in (or respectively out of) the frame, is 4805 bits in length (respectively 5084), thus the whole correcting rate $R$ is equal to 0.82 .

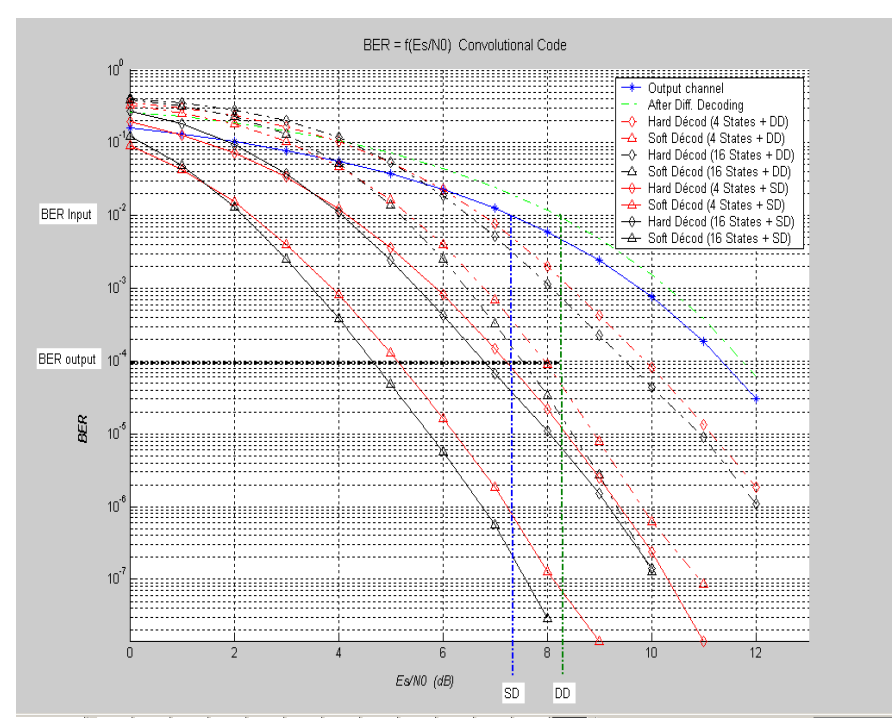

Figure 6. Convolutional Code 7,5 and 35,23 comparison

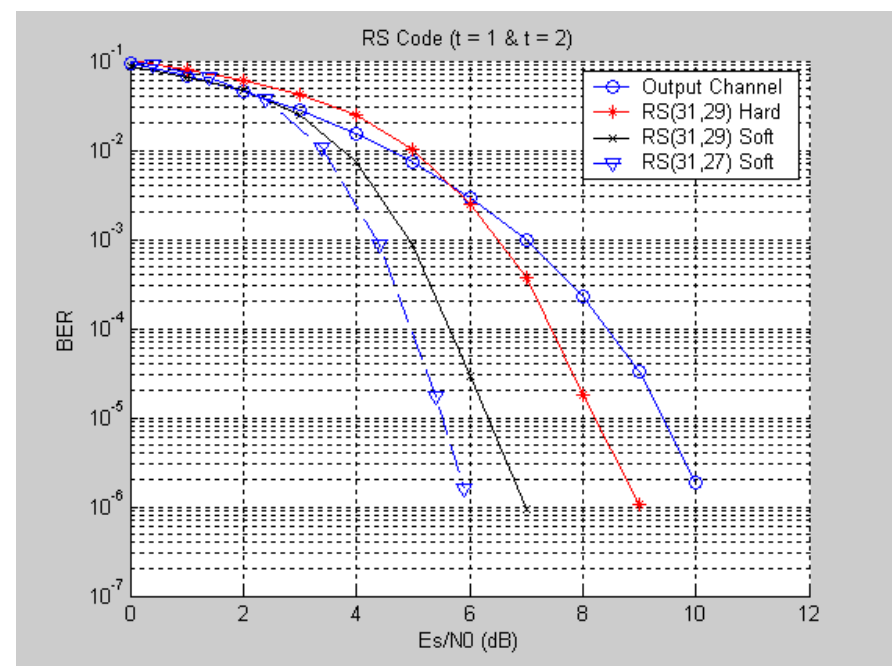

Figure 7. Reed Solomon $\operatorname{RS}(31,29)$ and $\operatorname{RS}(31,27)$ hard and soft decoding

\section{Simulation results}

Results presented are obtained by simulations in the presence of Additive White Gaussian Noise (AWGN). Fig. 6 presents performance obtained with $\mathrm{CC}$ coders (both for 4 and 16 states). It clearly appears that the use of a differential coding decreases performance in doubling the bit error rate. We take the BER at the channel output as a reference for the 2 options, with Differential Coding (DD) and without DD (SD). At $10^{-5}$, we have a loss of $2.5 \mathrm{~dB}$ when using DD both for hard and soft decoding. Let us recall that in the case of phase ambiguity, the decoding process may not work correctly. It also appears that we get better performance with the 16-state coder/decoder, but that 16-state decoding is more expensive in terms of computational load. All the configurations using the soft option 
make it possible to lower the BER from $10^{-2}$ to $10^{-4}$. In the case of RS coding/decoding (Fig. 7), the soft option does not allow us to reach a BER of $10^{-4}$. The use of RS-BTC $(31,29)$ (Fig. 8) lowers the BER values from $10^{-2}$ down to $10^{-4}$ after three iterations. RS-BTC provides a better code rate $(0.83)$ but a strong real-time constraint and a long transmitted frame.

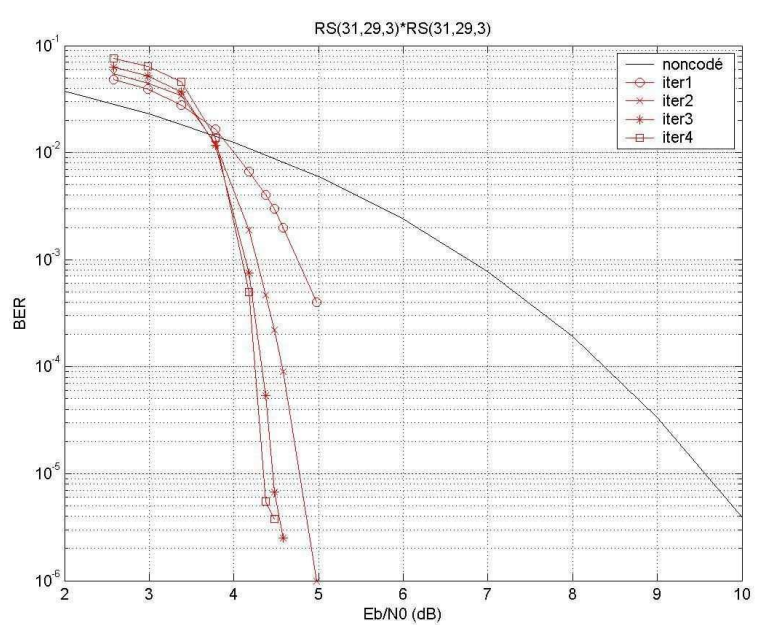

Figure 8. Reed Solomon Block Turbo Code $(31,29)$ soft decoding

\section{SEA TRIAL RESULTS}

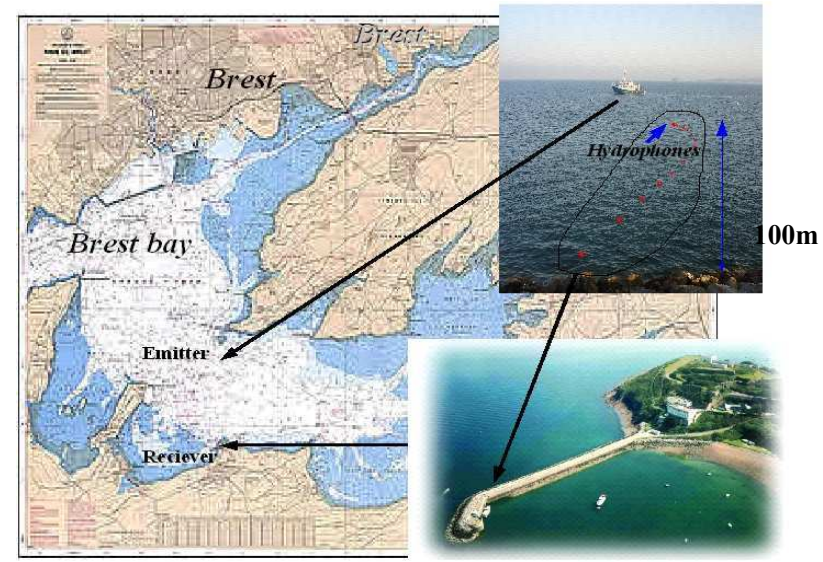

Figure 9. Sea trial site, Brest Bay

In order to test $\mathrm{CC}$ and $\mathrm{RS}$ channel coding, sea trials were carried out in the Bay of Brest in October 2007 (Fig. 9). This bay is a shallow underwater acoustic channel (10-50m depth). Objectives were to check both receiver and emitter operating in moving and realistic conditions. The emitter was on board a boat while the receiver was positioned in a laboratory van on the quay side. At reception, acoustic signals were received on four sensors. Hydrophone interspaces were almost $20 \mathrm{~cm}$ (around five wavelengths). The first hydrophone was deployed $100 \mathrm{~m}$ from the quay and at $5 \mathrm{~m}$ of depth. During the sea trials, 71 sequences of three minutes each were emitted, representing 3 hours and 33 minutes of recorded signals. QPSK modulation with two carrier frequencies $(11.2 \mathrm{kHz}$ or $17.5 \mathrm{kHz})$ was used. Demodulation, timing and carrier recovery and equalization were computed in real time in the receiver. Channel and source decoding were then performed to evaluate the transmission quality.

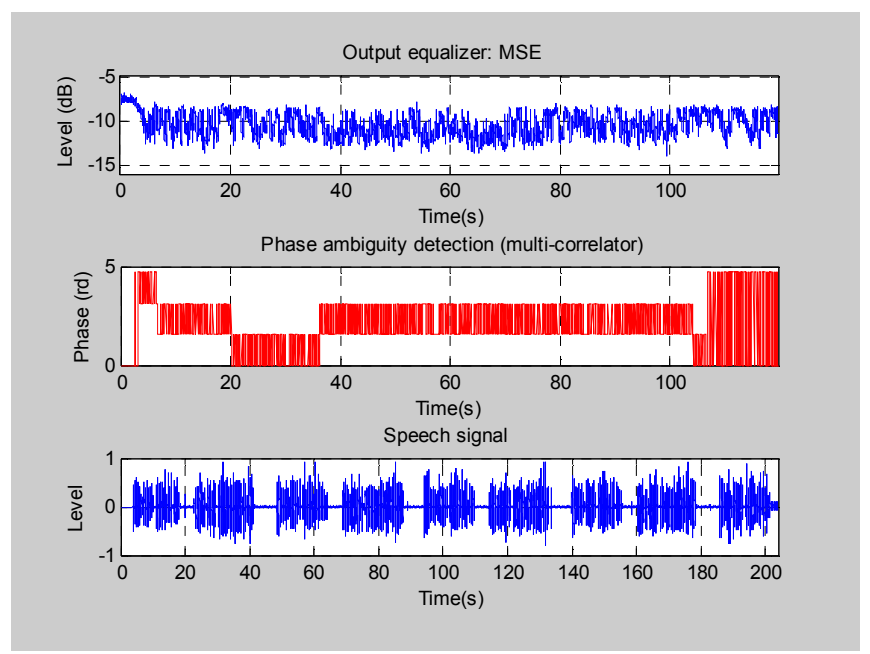

Figure 10. MSE, phase ambiguity, detection and Speech signal

One of the results concerns a speech signal transmission lasting 3 minutes. The carrier frequency is fixed at $17.5 \mathrm{kHz}$ and the bit rate is $5.8 \mathrm{kbps}$. The distance between the emitter and the receiver is $2500 \mathrm{~m}$. We can observe in Fig. 10 a large variation of the MSE at the output equalizer, due to UWA channel perturbation. These variations generate phase ambiguities, corrected by the phase detector. The speech signal is correctly decoded.

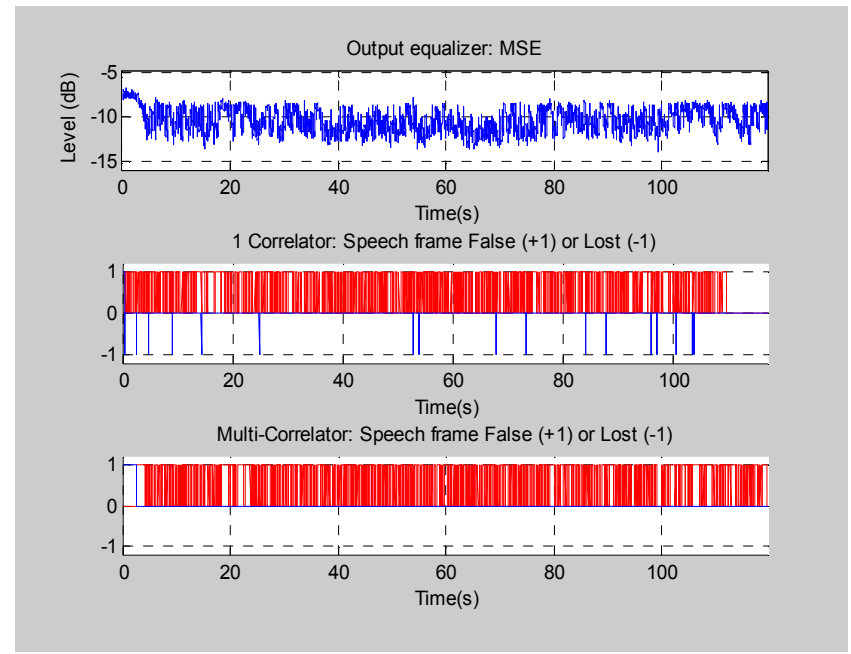

Figure 11. Frame false or lost with the use of one or five correlators

The information frame detected with the use of one or five correlators is shown in Fig. 11. The frame indicator equals +1 when the speech frames are false and -1 when the speech frames are lost. With one correlator, 125 frames are lost during the transmission, corresponding to $5.6 \mathrm{~s}$ of speech signal. With a multi-correlator, we do not lose any frames. After phase 
ambiguity correction and frame synchronization, the bit error rate fell from $4.0910^{-2}\left(\mathrm{BER}_{\text {in }}\right.$ decoder input) to $3.610^{-2}$ (BER ${ }_{\text {out }}$, output) in the case of hard decoding and $3.1410^{-2}$ in the case of soft decoding.

Other sea trials for testing RS-BTC channel coding took place in Brest Bay in March 2010. Two boats were used for these sea trials (Fig. 12). The transmitter was on board the "Aventurière II". The transducer was at a depth of $4 \mathrm{~m}$. The receiver was on board the boat "Idaco". The four hydrophones were towed behind the boat, $3 \mathrm{~m}$ below the keel. These trials were carried out in rough sea conditions. During the trials, five and a half hours of transmitted signal were recorded, representing 162 two-minute sequences. Both emitter and receiver were moving (less than 4 knots), with a range from $300 \mathrm{~m}$ up to $3000 \mathrm{~m}$. The position of the two boats during each sequence transmission (named AIT) for tests conducted on March 24 was recorded (Fig. 13). During these trials, three carrier frequencies and four bit rates for each carrier frequency were used to transmit the information frames.
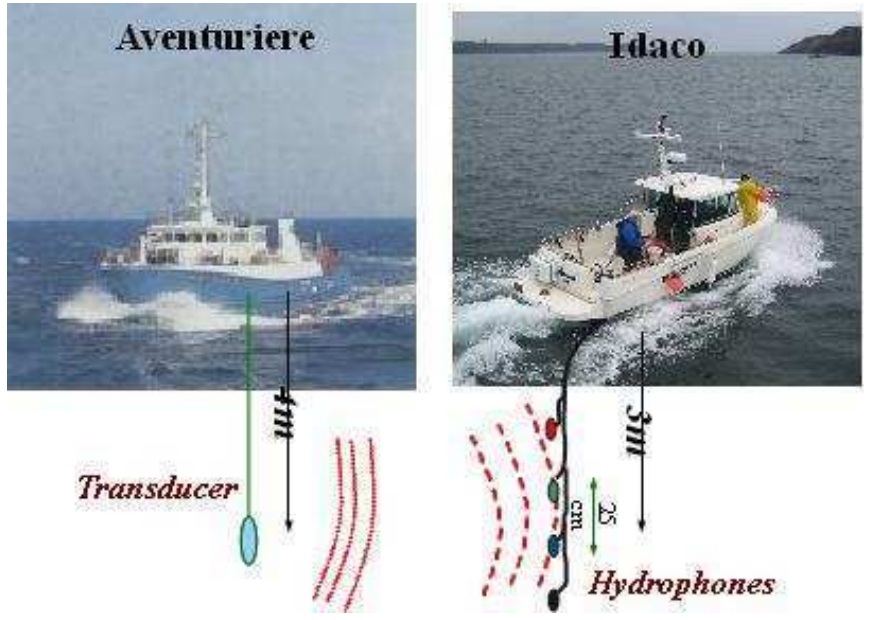

Figure 12. Sea trial configuration

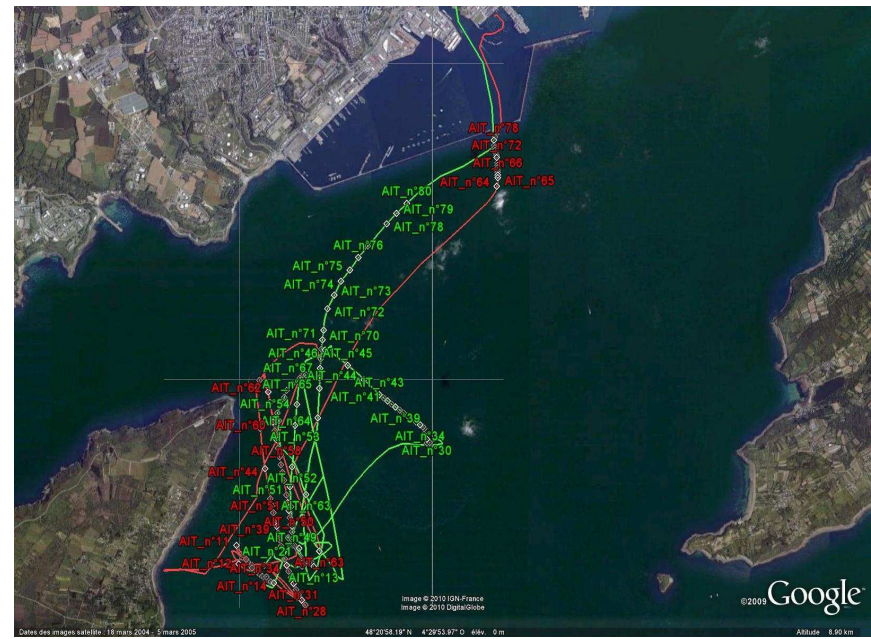

Figure 13. Brest Bay sea trials: recording position (March $24^{\text {th }}$ )
The result marked AIT24 shows the behavior of the receiver for image transmission with a $17.5 \mathrm{kHz}$ carrier frequency and a bit rate fixed at $11.6 \mathrm{kbps}$. Transmitter and receiver speeds are around 1 knot. The distance between the transmitter and the receiver was $1000 \mathrm{~m}$. In Fig. 14, the first plot shows the Mean Square Error (MSE). The success of the equalization process should be noted.

This sequence showed very good overall performance. It clearly appears that the transmission was processed without any kind of difficulty. This sequence was emitted without differential coding. After decoder synchronization, 8 iterations were processed for the channel decoding. The BER at the decoder input $\left(\mathrm{BER}_{\text {in }}\right)$ was $5.410^{-5}$ and 0.0 at the decoder output. For 238 frames transmitted, not a single frame was lost (second plot, Fig. 14), 6 (2.5\%) were detected false (one or more errors) at the channel decoder input (third plot, Fig. 14), and $0(0 \%)$ were detected false at the decoder output (fourth plot, Fig. 14). During the convergence stage, frames may be lost (only one incomplete frame during this trial). The seven images transmitted were correctly decoded.
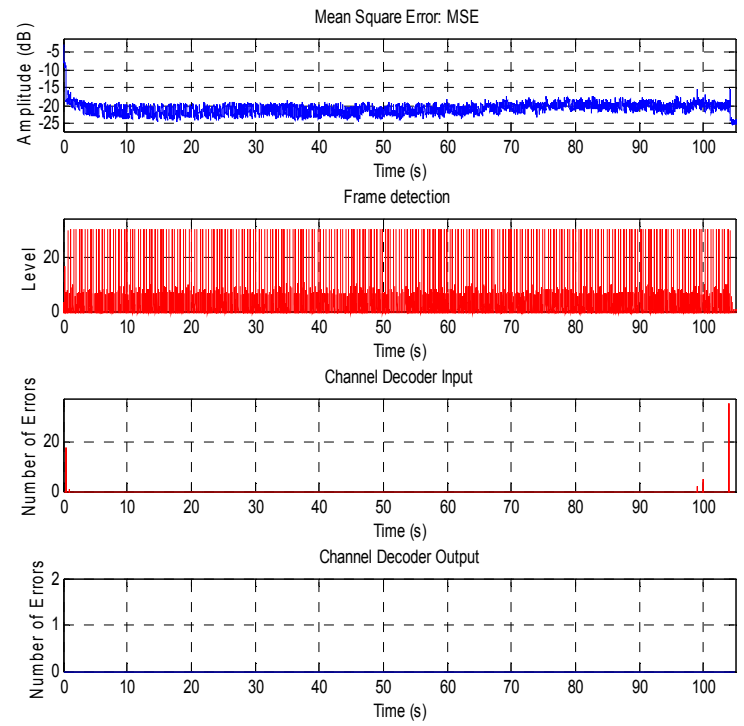

Figure 14. MSE, correlator output, decoder input and decoder output (AIT24)

The result noted AIT30 concerns a speech signal transmission. For this trial, the carrier frequency was $17.5 \mathrm{kHz}$ and the bit rate was set at $7.0 \mathrm{kbps}$, with $2.4 \mathrm{kbps}$ used for the speech signal. The distance between the transmitter and the receiver was $2500 \mathrm{~m}$. The MSE (first plot, Fig. 15) also shows how successful equalization was. After both phase ambiguity correction and decoder synchronization, the BER at the decoder input $\left(\mathrm{BER}_{\text {in }}\right)$ was $2.410^{-3}$ and the $\mathrm{BER}_{\text {out }}$ was 0.0 at the decoder output. For 144 frames transmitted, no frames were lost. 144 frames $(100 \%)$ were detected false at the decoder input, with $0(0.0 \%)$ false detections at the decoder output. Eight iterations were processed for the channel decoding. The speech signal was correctly decoded (Fig. 16). 

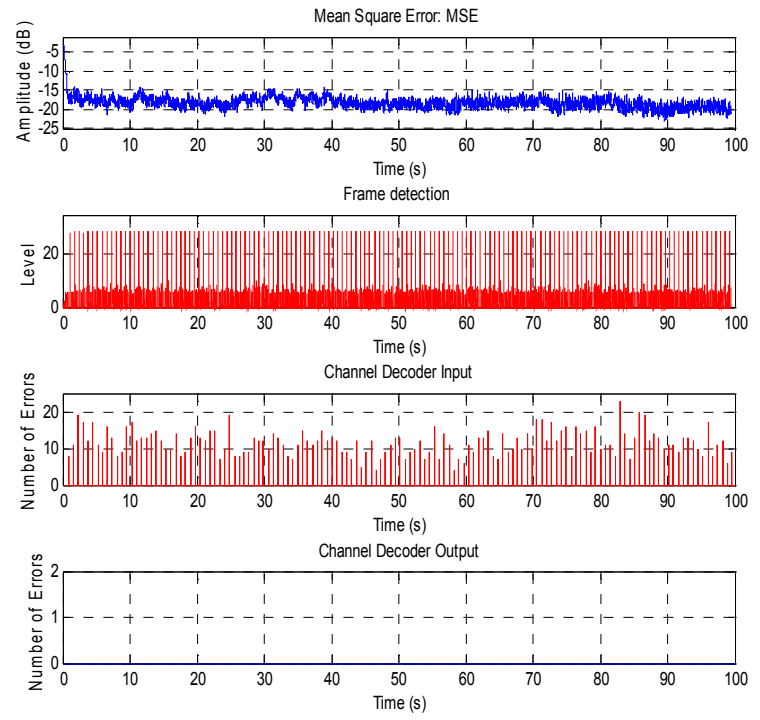

Figure 15. MSE, correlator output, decoder input and decoder output (AIT30)

The result indicated by AIT151 concerns image transmission. The distance between the transmitter and the receiver was $1000 \mathrm{~m}$. For this trial, the carrier frequency was $11.2 \mathrm{kHz}$ and the bit rate was fixed at $3.7 \mathrm{kbps}$. In this trial, the sequence was emitted without differential coding, so a phase jump appearing at the end of the transmission was not detected. The MSE (first plot, Fig. 17) also shows the equalization success, only disturbed during the phase jump. For 72 frames transmitted, 62 were detected, no frames were lost and 26 $(2.5 \%)$ were detected false at the decoder input, before the jump. The $\mathrm{BER}_{\text {in }}$ at the decoder input was $1.710^{-4}$ and the $\mathrm{BER}_{\text {out }}$ was 0.0 at the decoder output. 10 frames $(12 \mathrm{~s})$ were lost after the phase jump. Eight iterations were processed for channel decoding. The images were correctly decoded before the phase jump. A quarter of an image was lost during this trial.

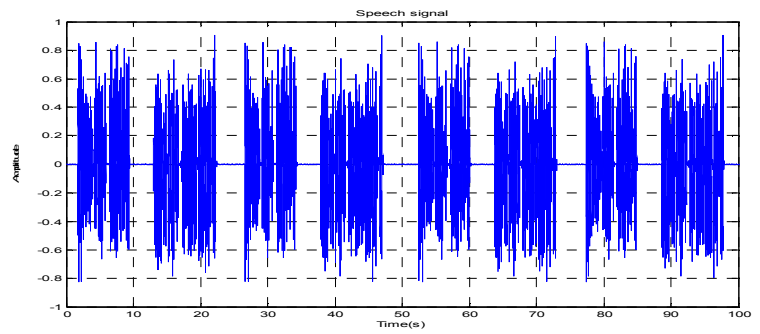

Figure 16. Speech signal (AIT30)

More than 5 hours of transmitted signal were recorded. A large number of sequences were successfully decoded at different distances, namely 300, 1000 and 2500m in moving conditions, using three carrier frequencies and different bit rates ranging from 3.7 to $11.6 \mathrm{kbps}$. These sequences show that channel coding/decoding perform well when synchronization and equalization work correctly.

\section{CONCLUSION AND PERSPECTIVES}

This paper presents the extension of the TRIDENT system, developed by GESMA in collaboration with TELECOM Bretagne. This underwater acoustic link is designed to transmit different kinds of data as text, images and speech signal. A blind spatio-temporal equalizer is used to reduce different shallow underwater acoustic perturbations. The first tests in an operational context show that real-time transmission of information (image, speech, text) is feasible even with harsh channels, such as the underwater acoustic channel, and in the presence of multiple interferers. In this context, the contribution of channel coding can improve the transmission robustness and protect the data transmitted from remaining errors. The aim is to lower the BER from $10^{-2}$ to $10^{-4}$. First, CC and RS channel coding were tested in real conditions. These two channel coding types do not provide an adequate level of correction. To improve underwater acoustic link performance, RS-BTCs were introduced and tested in real conditions. The use of RS-BTC allows us to reach a lower BER. CC and RS use a short frame length, whereas RS-BTC works on longer frames. Differential coding has been used to solve the phase ambiguities with the RS-BTC option in the case of phase jump and possible frame loss. RS-BTC requires a higher computational load but this is not a disadvantage for underwater acoustic communications due to the bit rate. CC and RS seem more suitable for speech signal transmission and RS-BTC for image transmission.

If the Doppler effect is not compensated for before any processing on the received signal, it is very difficult to extract information. Future work will be carried out to improve both Doppler-shift compensation and timing recovery. Due to impulsive noise, others interleavers have to be checked, especially the bits interleaver, in the case of RS-BTC.
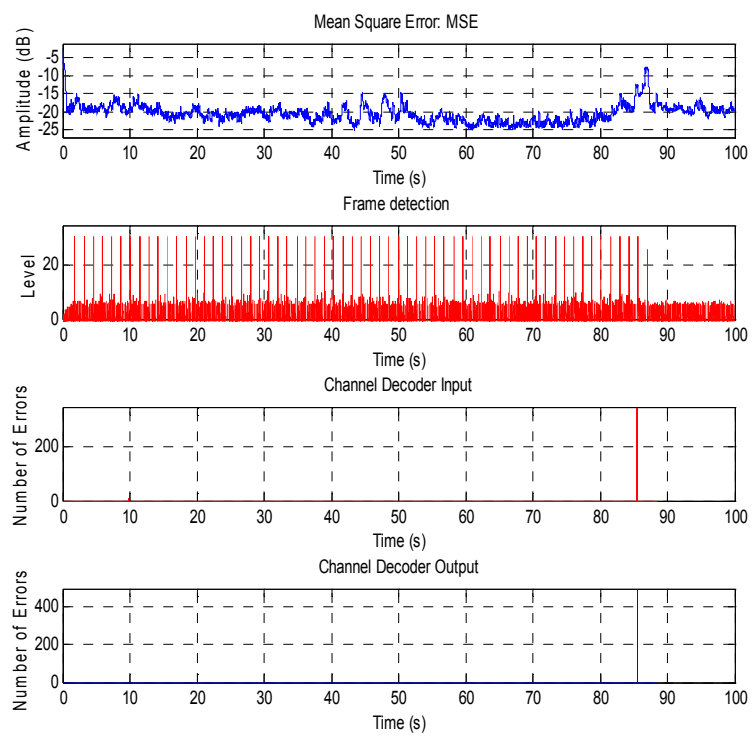

Figure 17. MSE, correlator output, decoder input and decoder output (AIT151) (undetected phase jump in the sequence) 


\section{References}

[1] J. Trubuil, G. Lapierre, T. Gall, J. Labat, "Real-time high data rate acoustic link based on spatio temporal blind equalization: the TRIDENT acoustic system," in Proc. OCEANS 2002, Biloxi, Vol.4, pp2438-2443.

[2] J. Labat and C. Laot, "Blind adaptive multiple input decision feedback equalizer with a self optimized configuration", IEEE trans on Comm, Vol. 49, N4, April 2001.

[3] A. Goalic, J. Trubuil and N. Beuzelin "Channel coding for underwater acoustic communication system", Oceans 2006, September 18-21, Boston, Ma, USA.

[4] J. Trubuil, A. Goalic and N. Beuzelin, "Synchronization and channel coding in shallow water acoustic communication", Oceans 2008, September 15-18, Québec, Canada.

[5] R. Pyndiah, A. Glavieux, A. Picart and S. Jacq, "Near optimum decoding of product codes", in Proc. Of IEEE Globecom '94, vol. 1/3, Nov.-Dec. 1994, pp.339-343.

[6] D. Chase. "A class of algorithms for decoding block codes with channel measurement information", IEEE trans. Inform. Theory, vol IT-8, Jan. 1972.

[7] E. R. Berlekamp, "Algebraic coding Theory", McGraw-Hill Book Company, New York, 1968 